10.30972/eitt.704762

\title{
Análisis didáctico de conocimientos previos sobre divisibilidad, de estudiantes que ingresan a la universidad usando como herramienta el 'Análisis Estadístico Implicativo'
}

\author{
Esp. Liliana N. Caputo; Dr. Ricardo R. F. Espinoza; Prof. Paula D. Bordón; \\ Mgter. Eduardo A. Porcel; Prof. María R. A. Ayala(*)
}

\section{Resumen:}

Este trabajo tiene por objetivo indagar sobre los saberes de divisibilidad de los estudiantes a través de sus respuestas a dos ítems de una evaluación mediante un análisis didáctico en el que se emplearon los resultados obtenidos por la herramienta Análisis Estadístico Implicativo (ASI). De este modo se quiere identificar definiciones, propiedades, procedimientos y argumentos y las relaciones conceptuales que se establecen entre estos objetos en las prácticas realizadas por los estudiantes.

ASI, a partir de la hipótesis de que, si un ítem a es más complejo que otro b, permite detectar relaciones del tipo "si a, entonces, casi siempre b", a las cuales se las denomina cuasi-implicaciones o reglas. Estas relaciones se presentan mediante un "grafo implicativo" para una mejor interpretación de los resultados obtenidos.

A modo general, se observaron relaciones de intensidades menores a 0.9 lo que permite afirmar que pocos estudiantes lograron establecer relaciones conceptuales que den cuenta de un alto grado de comprensión respecto a la divisibilidad de números enteros. 
Palabras clave: Comprensión de divisibilidad. Análisis Estadístico Implicativo. Cuasi-implicaciones.

\section{Introducción:}

Generalmente, las Instituciones de Educación Superior han vinculado los altos índices de fracaso de sus estudiantes en el primer año de las carreras de grado técnicas y vinculadas a las ciencias exactas, con deficiencias en la Escuela Secundaria (Nabarro et al, 2017).

Con el propósito de conocer los conocimientos previos con que los estudiantes ingresan al Nivel Superior y poder planificar la enseñanza de la Matemática a partir de ellos, se han realizado numerosas investigaciones a fin de explorar dichos saberes previos.

Entre dichos antecedentes merecen mencionarse los realizados por Carnelli, Catalano y Formica (2018); del Puerto, Minaard y Seminara (2006) y Abrate, Pochulu y Vargas (2006), consistentes en su mayoría en establecer los errores que cometen los estudiantes al resolver ciertos problemas o ejercicios y explicitar las posibles causas de dichos errores. Asimismo, desde 2000 el Grupo de Investigación de Matemática Aplicada a la Educación Educativa de la Facultad de Ciencias Exactas y Naturales y Agrimensura (FACENA) de la Universidad Nacional del Nordeste (UNNE), Argentina, se ha dedicado a indagar los conocimientos matemáticos previos de los ingresantes a las distintas carreras de la mencionada Casa de Altos Estudios; desde 2015, este grupo ha analizado pruebas diagnósticas de conocimientos matemáticos previos de ingresantes a la mencionada Facultad, utilizando Análisis Estadístico Implicativo (ASI), método de Estadística Multivariante que permite lograr esos objetivos, asociando de manera asimétrica los ítems de cuestionarios de evaluación.

En este trabajo, se presenta el análisis mediante dicha metodología de dos problemas referidos a divisibilidad de números enteros, correspondientes a la evaluación de conocimientos previos sobre dicho contenido suministrada a 181 ingresantes de las carreras Ingeniería Eléctrica, Ingeniería en Agrimensura e Ingeniería en Electrónica de FACENA, en 2019. 


\section{Marco teórico y metodológico}

El instrumento empleado para este trabajo pertenece a la Tesis Doctoral del Dr. Ricardo Fabián Espinoza (2019). En dicho trabajo, para elaborar tal instrumento se toman como marco de referencia los NAP 2011, los diseños curriculares, investigaciones enmarcadas en la Didáctica de la Divisibilidad y libros de texto de uso frecuente en las aulas, tanto de nivel medio como superior. Para cada uno de los problemas que forman parte del instrumento, se realiza un minucioso análisis usando las herramientas del Enfoque Ontosemiótico (EOS).

ASI permite descubrir reglas inductivas no simétricas para modelar relaciones entre variables. En principio, las mencionadas variables son los ítems de una evaluación y sus autores (Régis Gras y los miembros de su grupo de investigación de la Universidad de Nantes), partiendo de la hipótesis: "si un ejercicio es más complejo que otro, entonces todo alumno que resuelve el primero debería resolver también el segundo" (Régnier, 2013) crearon estas relaciones del tipo “si a, entonces, casi siempre b” (llamadas cuasi-implicaciones o reglas) entre las respuestas a dichos ítems.

Cabe aclarar que el término "un ejercicio es más complejo que otro" hace referencia al hecho de que, para resolverlo, se requieren saberes más avanzados o evolucionados que el otro.

En principio, la aplicación de ASI requiere de:

»Un conjunto finito de variables dicotómicas (V), donde a $\in \mathrm{V}$ asume el valor 1 si el ítem a ha sido resuelto correctamente y o en caso contrario.

» Un conjunto E, también finito, formado por todos los sujetos evaluados.

» Para cada par de variables a y b, los dos subconjuntos de E siguientes:

$$
\mathrm{A}=\{\mathrm{x} \in \mathrm{E} / \mathrm{a}(\mathrm{x})=1\} \text { y } \mathrm{B}=\{\mathrm{x} \in \mathrm{E} / \mathrm{b}(\mathrm{x})=1\}
$$

es decir, los conjuntos formados por los sujetos que respondieron correctamente los ítems a y b, respectivamente.

Ahora bien, según la Lógica clásica, para que la implicación $\mathrm{a} \Rightarrow \mathrm{b}$ sea verdadera, si a es verdadera, también lo debe ser $\mathrm{b}$ de donde debería ser $\mathrm{A} \subset \mathrm{B}$.

Sin embargo, en la práctica pueden darse casos de estudiantes que pertenecen a A, pero no a $\mathrm{B}$, sin que ello implique que no existe relación entre los ítems a y b. 
Para determinar la existencia o no de relación entre a y b, se relativizará el peso de los contraejemplos (los casos en que $\mathrm{a} \wedge \neg \mathrm{b}$ es verdadera que equivale a los $\mathrm{x} \in(\mathrm{A}-\mathrm{B}$ ) a partir de los cardinales de E, A, B y A - B.

Formalmente, se eligen dos subconjuntos X e Y de E (no se conoce a priori la existencia de vínculos entre ellos), coordinables con A y B, respectivamente.

La cuasi-implicación $\mathrm{a} \rightarrow \mathrm{b}$ es admisible al nivel de confianza 1 - $\alpha$ si, y sólo si, $\operatorname{Pr}[\mathrm{c}(\mathrm{X}-\mathrm{Y}) \leq \mathrm{c}(\mathrm{A}-\mathrm{B})] \leq \alpha$. (Gras y Kuntz; 2009)

"Estas probabilidades pueden calcularse utilizando las distribuciones Hipergeométrica, Binomial o de Poisson, según sea el tipo de población (finita o infinita) y el tamaño de muestra (fijo o indeterminado) (Bodin; 1995). Ahora bien, cuando el tamaño de la población (N) tiende a infinito, la Hipergeométrica tiende a la Binomial de parámetros n y p (siendo $\mathrm{n}$ el tamaño de muestra y p el cociente entre el número de casos favorables al suceso de interés y N), con p constante; pero - a su vez - cuando el tamaño de la muestra tiende a infinito, la Binomial converge a la ley de Poisson de parámetro $\lambda=\underline{\#(\mathrm{~A}) . \#(\mathrm{E}-\mathrm{B}) . ”(M e n d o z a}$ et. al, 2019)

$$
\#(\mathrm{E})
$$

Por otra parte, se define el índice de implicación como el número:

$$
q(a, \neg b)=\frac{\#(A-B)-\frac{\#(A) . \#(E-B)}{\#(E)}}{\sqrt{\frac{\#(A) \cdot \#(E-B)}{\#(E)}}}
$$

que estima la diferencia entre \# $(\mathrm{A}$ - B) y el valor que habría tomado si a y b fueran independientes (a la cual se denota con $Q(a, \neg b)$ ). Bajo determinadas condiciones $Q(a, \neg b)$ se aproxima a la distribución Normal (o,1), por lo que - a partir de q(a, $\neg$ b) - se define la intensidad de implicación, que mide la calidad inductiva de a sobre b, como sigue:

Luego, se puede redefinir la admisibilidad de $\mathrm{a} \rightarrow \mathrm{b}$ al nivel de confianza $1-\alpha$, diciendo que la misma es admisible a dicho nivel si $\varphi(a, b) \geq 1-\alpha$.

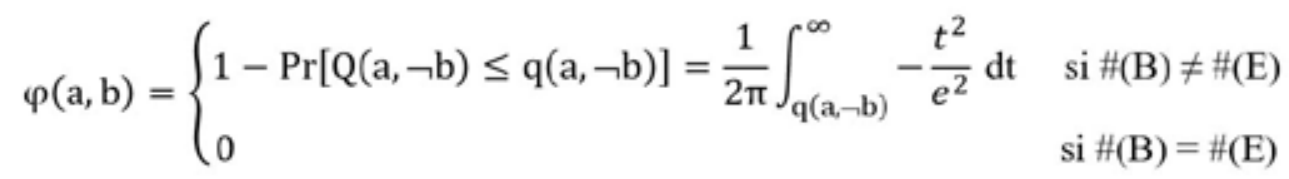


Ahora bien, cuando el cardinal de E es muy grande, se hace necesario considerar qué sucede con la calidad de la implicación contrarrecíproca, puesto que si \#(A) y \#(B) son pequeños con respecto a \#(E), los cardinales de sus complementos serán muy grandes. Es por eso que se incluye el concepto de entropía de Shannon que permite definir un índice que integra la información a partir de un número pequeño de contraejemplos de la regla $\mathrm{a} \rightarrow \mathrm{b}$ y de su contrarrecíproca.

Llamando $\alpha=\frac{\#(\mathrm{~A})}{\#(\mathrm{E})}, \beta=\frac{\#(\mathrm{E}-\mathrm{B})}{\#(\mathrm{E})} \mathrm{yt}=\frac{\#(\mathrm{~A}-\mathrm{B})}{\#(\mathrm{E})}, \quad$ se define la entropía de b habiéndose dado a, como sigue:

$$
\mathrm{h}_{1}(\mathrm{t})=\left\{\begin{array}{c}
\left.-\left(1-\mathrm{t} \alpha^{-1}\right) \log _{2}\left(1-\mathrm{t} \alpha^{-1}\right)-\mathrm{t} \alpha^{-1}\right) \log _{2} \mathrm{t} \alpha^{-1} \text { si } \mathrm{t} \in\left[0, \frac{\alpha}{2}[\right. \\
1 \text { si } \mathrm{t} \in\left[\frac{\alpha}{2}, \alpha\right]
\end{array}\right.
$$

De la misma manera, la entropía de $\neg$ a, si no se verifica b está dada por:

$$
h_{2}(t)=\left\{\begin{array}{c}
\left.-\left(1-t \beta^{-1}\right) \log _{2}\left(1-t \beta^{-1}\right)-t \beta^{-1}\right) \log _{2} t \alpha^{-1} \text { si } t \in\left[0, \frac{\beta}{2}[\right. \\
1 \text { si } t \in\left[\frac{\beta}{2}, \beta\right]
\end{array}\right.
$$

Así pues, el índice mencionado es $\quad \mathrm{i}(\mathrm{a}, \mathrm{b})=\sqrt[4]{\left(1-\mathrm{h}_{1}^{2}(\mathrm{t})\right)\left(1-\mathrm{h}_{2}^{2}(\mathrm{t})\right.}$.

Finalmente, se define la intensidad entrópica de $\mathrm{a} \rightarrow \mathrm{b}$ mediante el número:

$$
\psi(a, b)=\sqrt{i(a, b) \cdot \varphi(a, b)}
$$

Para calcular las intensidades de una implicación (clásica o entrópica) se utiliza el software Classification Hiérarchique Implicative et Cohésitive (CHIC), desarrollado originalmente por R. Gras y H. Rostam.

CHIC proporciona una tabla de doble entrada que relaciona todas las variables, pero que dificulta interpretar cuáles son las implicaciones que se dan entre ellas. Por tal motivo, el soft presenta las cuasi-implicaciones de mayor intensidad en un digrafo ponderado, sin ciclos, que se denomina grafo implicativo, y que facilita la visualización y comprensión de las principales relaciones obtenidas al hacer el análisis. (Couturier, 2009)

Si bien en la Lógica clásica la proposición ( $\mathrm{a} \Rightarrow \mathrm{b} \Rightarrow \mathrm{b} \Rightarrow \mathrm{c}) \Rightarrow(\mathrm{a} \Rightarrow \mathrm{c}$ ) es una tautología (Ley del Silogismo Hipotético), cuando se trabaja con las cuasi-implicaciones definidas 
anteriormente, dicha implicación no es necesariamente una ley de inferencia. Para que ello ocurra, debe ser $\psi(\mathrm{a}, \mathrm{c}) \geq 0,5$

\section{PROBLEMAS ANALIZADOS}

Problema 1: Responde las siguientes preguntas, fundamentando cada respuesta.
a) ¿3 es divisor de 30 ?
b) ¿3 es divisor de 473 ?
c) ¿3 es factor de 30 ?
d) ¿441 es múltiplo de 7 ?

Problema 5: Teniendo en cuenta que: 187 = 11x17, ¿̇son correctas las siguientes afirmaciones?:
a) 17 es divisor de $11 \times 17$.
b) 11x17+1870 es múltiplo de 187 .
c) $11 \times 17+16$ es múltiplo de 187 .
En cada caso, fundamenta tu respuesta.

Estos problemas son similares dado que las tareas involucradas son las mismas, sin embargo en ambos casos los números están expresados de manera diferente como puede verse en el análisis a priori realizado para validar el cuestionario (Espinoza et al, 2019). En efecto, en el ejercicio 5 , si bien se dice que $11 \times 17=187$, en cada ítem en vez de 187 se usa 11x17, involucrando saberes más complejos que en el ejercicio 1 (por ejemplo, en el ítem $5 \mathrm{c}$ la unicidad del cociente y el resto en la división entera).

\section{Resultados}

Al usar CHIC para obtener las cuasi-implicaciones admisibles y sus intensidades, se obtuvo el grafo implicativo de la Figura 1.

En el mismo, se puede observar que las mayores intensidades corresponden a las reglas $5 \mathrm{c} \rightarrow 1 \mathrm{~d}$ y $1 \mathrm{~b} \rightarrow 1 \mathrm{a}$.

Se observa que el grafo admite dos vértices iniciales (1c y 5c) y uno terminal (1a).

Así pues, puede asegurarse que los dos ítems que resultaron de mayor dificultad a los ingresantes evaluados son los ítems 1c (¿̇3 es factor de 30?) y 5c (¿்11x17 + 16 es múltiplo de 187?); en cambio, el problema que les resultó más sencillo fue decidir y justificar si 3 es un divisor de 30 (1a). 


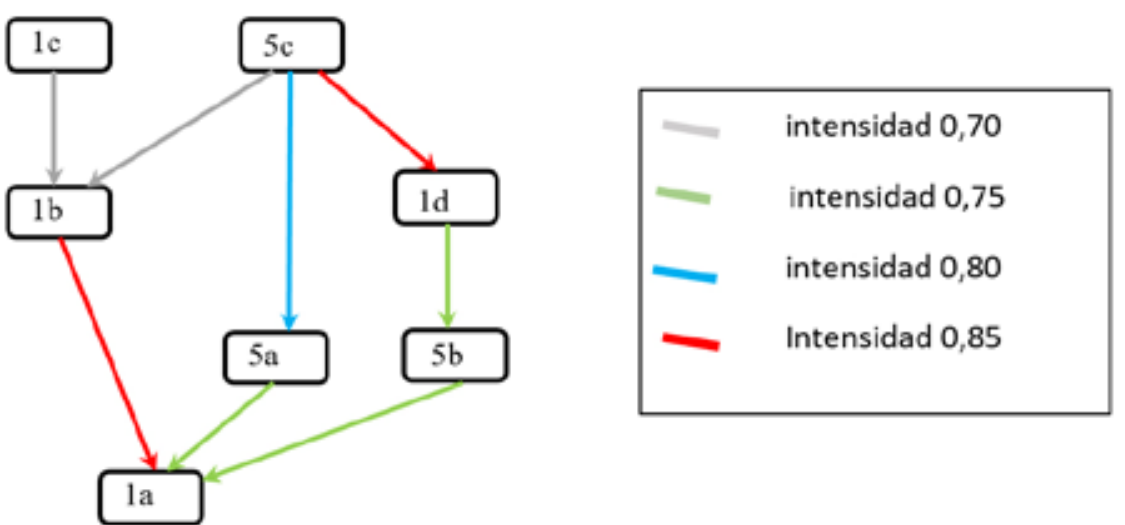

Figura 1: Grafo implicativo

Estos primeros resultados se corresponden con el análisis de frecuencias de las respuestas que se presentan en la Tabla 1, en la que puede observarse que los menores porcentajes de respuestas correctas corresponden a los ítems 1c y 5c (señalados en rojo), así como también que el ítem 1a fue el que obtuvo el mayor porcentaje de respuestas correctas (resaltado en azul).

Tabla 1. Estadística descriptiva de las respuestas a los siete ítems analizados

\begin{tabular}{|c|c|c|c|}
\hline Items & $\begin{array}{c}\% \text { de } \\
\text { respuestas }\end{array}$ & $\begin{array}{c}\% \text { de respuestas } \\
\text { correctas }\end{array}$ & $\begin{array}{c}\text { Correctas sobre el } \\
\text { total de evaluados (\%) }\end{array}$ \\
\hline 1a & 97 & 75 & 75 \\
\hline 1b & 92 & 63 & 63 \\
\hline 1c & 71 & 45 & 45 \\
\hline 1d & 83 & 60 & 60 \\
\hline 5a & 83 & 56 & 56 \\
\hline 5b & 80 & 61 & 61 \\
\hline 5c & $\mathbf{7 8}$ & $\mathbf{4 7}$ & $\mathbf{4 7}$ \\
\hline
\end{tabular}

Se atribuye el bajo porcentaje de respuestas correctas al ítem 1c a que los estudiantes desconocen el uso del término factor como sinónimo de divisor.

El bajo porcentaje de respuestas correctas al ítem $5 \mathrm{c}$ puede deberse a la dificultad de reconocer a un número (aun cuando no se sabe cuál) como a un posible dividendo expresado en función de su cociente (1) y de su resto (16) en la división entera por 187.

Si bien tanto en los ítems 5b y 5c se solicita determinar si un número es múltiplo de otro, los problemas son diferentes dado que en $5 \mathrm{~b}$ el número está expresado como la suma de un 
producto por un número y en el 5 c en función del algoritmo de la división; por ello, los saberes puestos en juego en cada uno de los problemas son diferentes, aunque en ambos se solicite determinar si los números son múltiplos de 187.

Para ambos ítems los estudiantes pudieron emplear la calculadora para resolver la cuenta y luego dividir por 187. Como en $5 \mathrm{~b}$ el valor que muestra la calculadora es un número entero pudieron interpretar que el número es un múltiplo de 187, en cambio en $5 \mathrm{c}$ el número devuelto por la calculadora no es entero y posiblemente no hayan podido decidir si se trata o no de un múltiplo de 187.

Se presenta a continuación el análisis de las cuasi implicaciones cuyas intensidades superan 0,75 . Se señala para cada una de ellas algunas relaciones establecidas por los estudiantes y algunos de los argumentos más relevantes que las justifican. En total son tres: $\mathbf{5} \mathbf{c} \rightarrow \mathbf{1 d}$; $\mathbf{1 b}$ $\rightarrow \mathbf{1 a}$ con una intensidad de 0,85 y $5 \mathbf{c} \rightarrow \mathbf{5 a}$ con una intensidad de 0,80 .

Para interpretar la relación entre $\mathbf{5 c}$ y $\mathbf{1 d}$, hay que considerar que, en 1d, el dividendo es 441, el divisor es 7, el cociente es 157 y el resto es cero. Por ello, los estudiantes que respondieron $5 \mathbf{c}$ correctamente y pudieron justificar su valor de verdad, pudieron hacerlo en el caso de $\mathbf{1 d}$.

A partir de la lectura de las respuestas de los estudiantes es notable que muchos de ellos han realizado las operaciones y expresado en forma decimal al número 11x17+16 y así logran emplear los mismos procedimientos para resolver ambos ítems.

Este modo de proceder coincide con lo señalado por Bodí, Valls y Llinares (2009) respecto a alumnos del nivel secundario: "Mayoritariamente necesitan obtener la representación decimal del número y dividir para discutir la divisibilidad...”, a situaciones similares hacen referencia otros investigadores como Zazkis y Gadowsky (2001) y Brown (2002).

El sentido de la cuasi implicación se fundamenta en que la tarea $5 \mathrm{c}$ requiere determinar que un número no es un múltiplo de otro, mientras que $1 \mathrm{~d}$ sí lo requiere, puesto que como las definiciones se formulan usando afirmaciones que caracterizan a un objeto y no las que no lo caracterizan, resulta más complejo determinar cuándo un número no es múltiplo de otro que cuando sí lo es. 
Como se ha ya mencionado, en el grafo implicativo se evidencia que por cada ítem es posible establecer una cuasi-implicación que tenga por consecuente a 1a. Esto se debe a que es muy sencillo y puede ser resuelto a partir de una gran variedad de estrategias por parte de los estudiantes.

En particular, en la relación entre $\mathbf{1 b}$ y $\mathbf{1 a}$ puede pensarse que los estudiantes que reconocieron que 3 es divisor de un número grande, pueden distinguir que 3 es divisor de un número más pequeño, como 30. También puede pensarse que si pueden emplear el criterio de divisibilidad por 3 para reconocer que un número no es divisible por 3 pueden emplearlo para reconocer que 30 es divisible por 3.

En la relación entre $5 \mathbf{c}$ y $5 \mathbf{a}$ vuelve ponerse en evidencia que los estudiantes que saben reconocer correctamente que un número no es múltiplo de otro saben reconocer cuándo sí lo es y en particular permite pensar que:

- Saber reconocer que cuándo un número expresado en base al algoritmo de la división no es un múltiplo de otro implica reconocer cuándo un número no es múltiplo si ambos están expresados en versión decimal.

- Saber que un número no es múltiplo de otro implica saber cuándo sí lo es.

- Saber que un número es múltiplo de otro implica saber que un número es divisor de otro (considerando que los números están en su versión posicional en base 10, pues en el $5 \mathbf{a}$ resuelven la cuenta 11x17 y después dividen para saber si es divisor.

\section{Consideraciones finales}

Teniendo en cuenta el sentido de las cuasi-implicaciones detectadas mediante ASI se pone en evidencia que si los estudiantes pueden determinar que un número no es múltiplo de otro saben reconocer cuándo sí lo es al igual que si saben reconocer que un número no es divisor de otro saben reconocer cuando sí lo es.

Sin embargo, las cuasi-implicaciones detectadas no son demasiado fuertes, lo cual permite afirmar que los alumnos evaluados no han logrado establecer relaciones conceptuales que den cuenta de un alto grado de comprensión respecto a la divisibilidad de números enteros; por ello, los resultados obtenidos constituyen un desafío para los docentes de los primeros cursos de Matemática de las carreras de Ingeniería de FACENA, el de diseñar y 
llevar al aula actividades de enseñanza que coadyuven a los estudiantes a fortalecer las definiciones, propiedades, procedimientos, argumentos y sus relaciones sobre divisibilidad.

\section{REFERENCIAS BIBLIOGRAFICAS}

Abrate, R.; Pochulu, M.; Vargas, J. (2006). "Errores y Dificultades en Matemática. Análisis de causas y sugerencias de trabajo”. Buenos Aires, Argentina: Universidad Nacional de Villa María.

Bodí, S.; Valls, J.; Llinares, S. (2009). La comprensión de la divisibilidad en N. Un análisis implicativo. En teoría y aplicaciones del Análisis Estadístico Implicativo. Primera aproximación en lengua hispana. Orús, P.; Zamora, L.; Gregori, P. (Eds). Santiago de Cuba, Cuba: Universitat Jaume I de Castellón y Universidad de Oriente. 215 - 233.

Bodin, A. (1995). “Analyse implicative: Modèles sous-jacents à l'analyse implicative et outils complémentaires”. Publications de l'Institute de Recherche Mathématiques de Rennes, fascicule 3 Fascicule de Didactique De Mathématiques I’E.I.A.O. $1-23$.

Carnelli, G.; Catalano, L.; Formica, A. (2018). “Diseño y estudio de resultados de una evaluación estructurada en un curso de Matemática con estudiantes que ingresan a la universidad”. Yupana Revista de Educación Matemática de la Universidad Nacional del Litoral No $10.16-29$.

Brown, A. (2002). "Patterns of thought and prime factorization". En S. R. Campbell y R. Zazkis (Eds.), Learning and teaching number theory: Research in cognition and instruction. Westport, CT: Ablex Publishing. 131-137. 
Couturier, R. (2009). “CHIC: utilización y funcionalidades. En Teoría y aplicaciones del Análisis Estadístico Implicativo”. Primera aproximación en lengua hispana. Orús, P.; Zamora, L.; Gregori, P. (Eds). Santiago de Cuba, Cuba: Universitat Jaume I de Castellón y Universidad de Oriente. 51 - 64.

Del Puerto, S.; Minnaard, C.; Seminara, S. (2006). “Análisis de los errores: una valiosa fuente de información acerca del aprendizaje de las Matemáticas”. Revista Iberoamericana de Educación 38(4). 1 - 13.

Espinoza, R. (2019). "La comprensión alcanzada por estudiantes de Profesorado en Matemática, referida a la Divisibilidad, al comenzar la Universidad”. Facultad de Humanidades y Ciencias Sociales, Universidad Nacional de Misiones. Disponible en: http://enfoqueontosemiotico.ugr.es/tesis/Tesis_RFEspinoza_2018.pdf

Espinoza, R.; Caputo, L.; Bordón, P.; Ayala, M.; Porcel, E. (2019). “Análisis a priori de un instrumento de evaluación sobre divisibilidad de números enteros con Análisis Estadístico Implicativo". Cadernos do IME. Universidade de Rio de Janeiro. v. 46. 1 - 15.

Gras, R.; Kuntz, P. (2009). "El Análisis Estadístico Implicativo (ASI) en respuesta a problemas que le dieron origen”. En Teoría y aplicaciones del Análisis Estadístico Implicativo. Primera aproximación en lengua hispana. Orús, P.; Zamora, L.; Gregori, P. (Eds). Santiago de Cuba, Cuba: Universitat Jaume I de Castellón y Universidad de Oriente. 3 - 50.

Mendoza, M.; Caputo, L.; Porcel, E.; Bordón, P. (2019). “Conocimientos previos sobre propiedades de operaciones con números reales de ingresantes a la Universidad. Su análisis usando Análisis Estadístico Implicativo”. Revista de la Escuela de Perfeccionamiento en Investigación Operativa No $46.42-53$.

Nabarro, S.; Ibarra, E.; Cejas, C.; Ger, S. (2017). "Las prácticas educativas y el desarrollo de competencias básicas en las carreras de la Facultad de Ciencias Forestales”. Libro de Actas del XII Encuentro Internacional de Educación Matemática en carreras de Ingeniería, pp 183 - 189. Santiago del Estero: U.N. de Santiago del Estero. ISBN 978-987-720-151-2. 
Régnier J-C. (2013). Extracto de la obra "Analyse Statistique Implicative. Une méthode d'analyse de données pour la recherche de causalités”. Gras, R.; Régnier J-C.; Guillet F. (Eds) (2009). Disponible en: http://sites.univ-lyon2.fr/asi7/?page $=$ o\&lang $=$ es.

Zazkis, R. \& Gadowsky, K. (2001). "Attending to transparent features of opaque representations of natural numbers". In A. Cuoco (Ed.), NCTM 2001 Yearbook: The roles of representation in school mathematics. Reston: NCTM. 41-52. 